\title{
Tratamento cirúrgico da fratura de mandibula atrófica pela técnica AO: relato de caso
}

\author{
Atrophic mandible fracture management by AO technique: case report \\ El Tratamiento Quirúrgico de la Fractura de Mandíbula Atrófica técnica AO: \\ reporte de caso \\ Rodrigo dos Santos PEREIRA ${ }^{1}$ \\ João Paulo BONARDI ${ }^{2}$ \\ Jonathan Ribeiro da SILVA ${ }^{2}$ \\ Carlos Fernando de Almeida Barros MOURÃO ${ }^{3}$ \\ Paulo Roberto BARBOSA JÚNIOR ${ }^{1}$ \\ Luís Fernando MAGACHO ${ }^{1}$
}

${ }^{1}$ Hospital Geral de Nova Iguaçu, Serviço de Cirurgia Bucomaxilofacial, 26041-050 Nova Iguaçu - RJ, Brasil

${ }^{2}$ Faculdade de Odontologia de Araçatuba, Universidade Estadual Paulista, UNESP, 16015-050, Araçatuba - SP, Brasil

${ }^{3}$ Faculdades São José, Departamento de Cirurgia Bucomaxilofacial, 21710-232 Rio de Janeiro, RJ, Brasil

\begin{abstract}
Resumo
As fraturas das mandíbulas atróficas são de difícil tratamento, pois necessitam de um vasto conhecimento por parte do cirurgião maxilofacial. Como é uma injuria incomum, muitos profissionais não possuem a experiência necessária para trata-las. O objetivo deste artigo é demonstrar um caso clínico onde foi empregada a técnica cirúrgica errada para tratar uma fratura de mandíbula atrófica bilateral e seu retratamento através da filosofia preconizada pela AO Foundation empregando uma placa de reconstrução $2.4 \mathrm{~mm}$ e enxertia com osso autógeno particulado da crista ilíaca anterior. Após 1 e 7 meses conclui-se que, apesar de ser uma técnica mais agressiva, proporciona os resultados mais satisfatórios e previsíveis.

Descritores: Fixação Interna de Fraturas; Técnicas de Fixação da Arcada Osseodentária; Arcada Edêntula.
\end{abstract}

\begin{abstract}
Fractures of atrophic mandibles are difficult to resolve because requires a large knowledge by oral \& maxillofacial surgeons. As it is an unusual injury, many surgeons don't have experience to treat them. The aim of this article is to report a clinical case where a wrong technique to fix the mandible was used and its treatment by AO Foundation philosophy with a recon $2.4 \mathrm{~mm}$ plate and the grafted with autogenous bone graft from anterior crest of iliac bone. After 1 year and 7 months we concluded that it is an aggressive technique but provides a satisfactory and predictable outcome.

Descriptors: Fracture Fixation, Internal; Jaw Fixation Techniques; Jaw, Edentulous.
\end{abstract}

\begin{abstract}
Resumen
Las fracturas de los maxilares atróficos son difíciles de tratar debido a que necesitan un amplio conocimiento por parte del cirujano maxilofacial. ¿Cómo es una lesión poco frecuente, muchos profesionales no tienen la experiencia necesaria para tratarlos? El propósito de este artículo es mostrar un caso en el que se empleó la técnica quirúrgica equivocada para tratar una fractura de mandíbula atrófica bilateral y la repetición del tratamiento a través de la técnica recomendada por la Fundación AO empleando una placa de reconstrucción de $2,4 \mathrm{~mm}$ y el injerto con partículas de hueso autógeno de la cresta ilíaca arriba. Después de 1 y 7 meses han llegado a la conclusión de que, a pesar de una técnica más agresiva proporciona los resultados más satisfactorios y predecibles.

Descriptores: Fijación Interna de Fracturas; Técnicas de Fijación de Maxilares; Arcada Edéntula.
\end{abstract}

\section{INTRODUÇÃO}

O tratamento da fratura mandibular possui o objetivo de reestabelecer a anatomia óssea, devolver a função, as condições estéticas além de ter evoluído desde o tratamento com bloqueio maxilomandibular até a redução aberta e a fixação com placas e parafusos ${ }^{1,2}$. O maior resultado da técnica de fixação interna rígida foi eliminar o período de bloqueio maxilomandibular por 6 semanas, permitindo que o paciente tenha o imediato retorno as suas funções.

A escolha do tratamento dependerá das características das fraturas e das condições clínicas do paciente. Os diferentes sistemas de placas e parafusos utilizados na região maxilofacial podem ser classificados em "Load Shearing" ou carga compartilhada e "Load Bearing" ou carga suportada". As fraturas simples ou lineares de pacientes dentados são tratadas utilizando placas "Load Shearing", porém, os pacientes que apresentarem fraturas cominutivas ou atróficas utiliza-se placas mais robustas do sistema "Load Bearing".

Uma condição clínica comum nos pacientes idosos é o edentulismo que leva perda óssea progressiva, diminuição da vascularização, resultando em um osso mais denso ${ }^{3}$. Luhr et al. classificou as mandíbulas edêntulas conforme a altura óssea remanescente. Classe I aquelas que apresentam com altura remanescente entre 16 e $20 \mathrm{~mm}$, classe II entre 11 e 15 $\mathrm{mm}$ e classe III menor ou igual a $10 \mathrm{~mm}^{4}$.

As fraturas em mandíbulas atróficas são incomuns, com 2,3\% de incidência e a maioria dos cirurgiões não possuem experiência no tratamento dessas injúrias ${ }^{3,5}$. As condições anatômicas presentes nas mandíbulas atróficas não permitem a utilização das placas "Load Shearing" pois, as linhas de tensão (na borda superior da mandíbula) e compressão (na borda inferior) se aproximam diminuindo a possibilidade óssea de compartilhar carga. A AO Foundation recomenda o uso de placas do sistema $2.4 \mathrm{~mm}$ RECON com parafusos ancorados nos ramos e sínfise para fixar as fraturas em mandíbulas atróficas ${ }^{6}$. Este sistema proporciona estabilidade suficiente para o reparo da fratura e a vascularização dos fragmentos ósseos através do suporte da carga funcional nas áreas de fratura. 
O objetivo deste artigo é demonstrar um caso clínico de fratura de mandíbula atrófica tratada erroneamente e a correção utilizando uma placa de reconstrução do sistema $2.4 \mathrm{~mm}$ e enxerto ósseo autógeno da crista ilíaca anterior.

\section{CASO CLÍNICO}

Paciente do gênero feminino, 82 anos, compareceu ao ambulatório de Cirurgia e Traumatologia Bucomaxilofacial do Hospital Geral de Nova Iguaçu - RJ referindo queixas álgicas em ambas as articulações temporomandibulares (ATM) e desvio acentuado da mandíbula para o lado direito após uma cirurgia para redução e fixação de uma fratura mandibular em outro serviço (Figura 1).

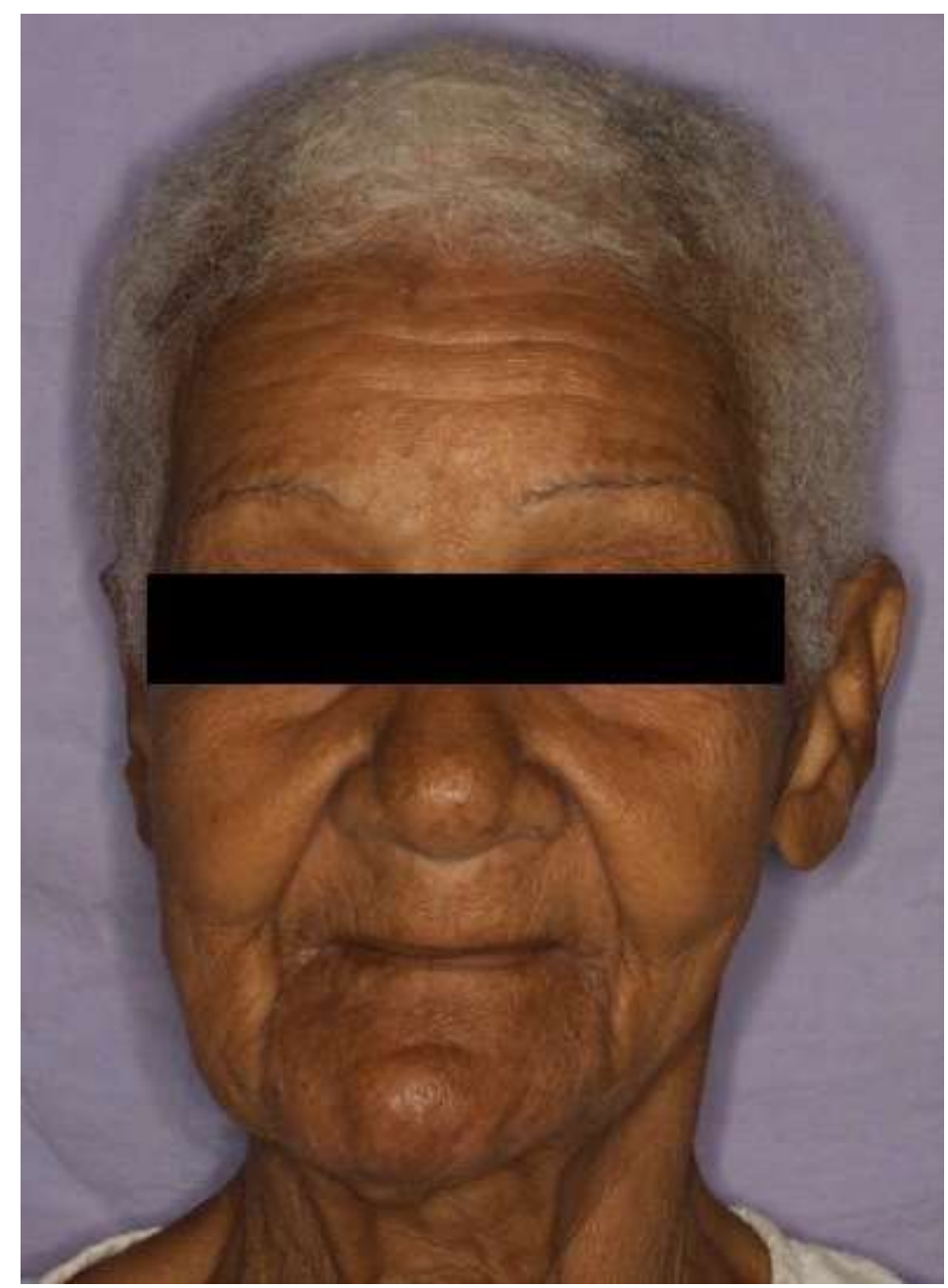

Figura 1: Fotografia clínica da paciente apresentando desvio mandibular à direita.

A paciente apresentava uma radiografia panorâmica realizada após o procedimento cirúrgico onde se observou uma mandíbula atrófica classe III de $\mathrm{Luhr}^{4}$ fixada em ambos os lados com: uma placa de perfil mais largo e parafusos bicorticais no lado direito e, no lado esquerdo, um fio de aço transpassando a região parassinfisária além de uma placa de perfil mais fino também fixada com parafusos bicorticais (Figura 2).

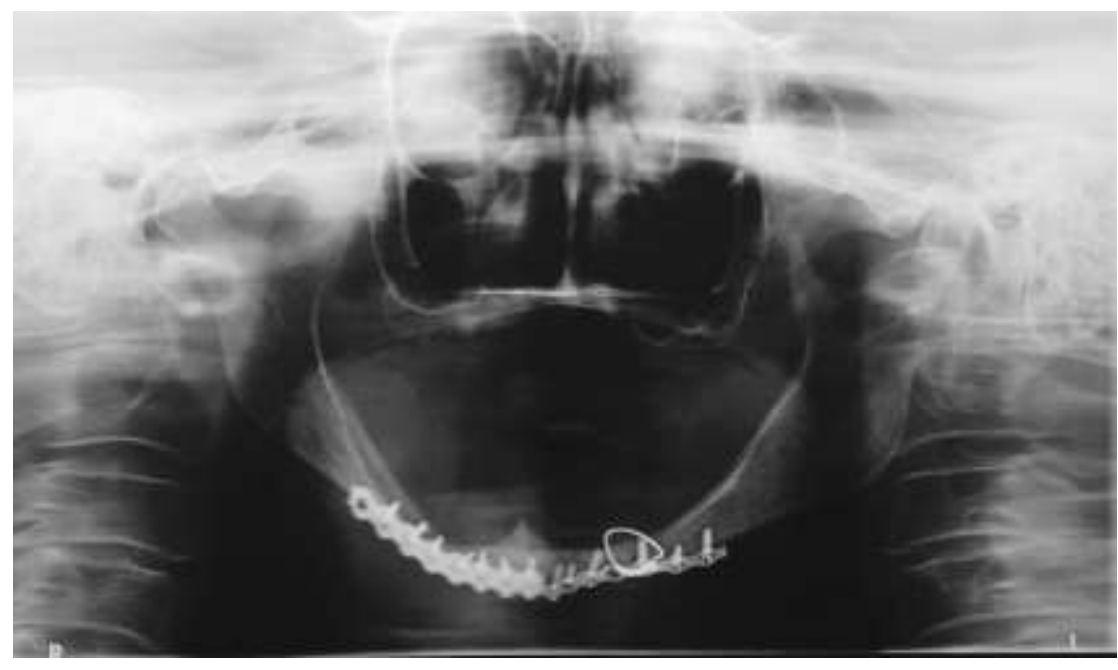

Figura 2: Fotografia da radiografia panorâmica demonstrando a fixação realizada previamente para o tratamento das fraturas mandibulares.
A tomografia computadorizada (TC) solicitada pela equipe demonstrou alteração posicionamento condilar e torção no côndilo mandibular direito (Figuras 3 e 4).

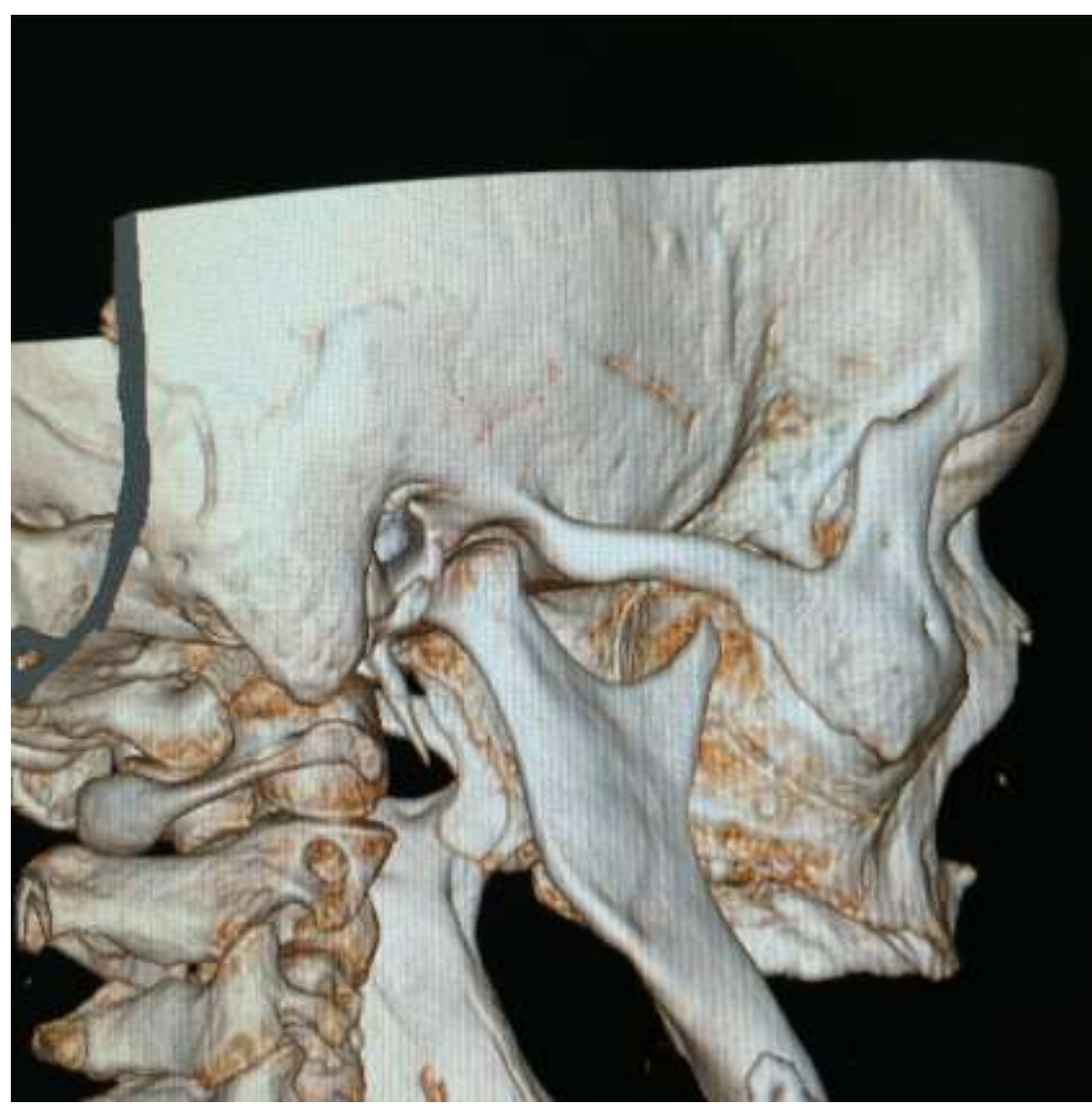

Figura 3: Reconstrução da tomografia computadoriza evidenciando torção condilar direita.

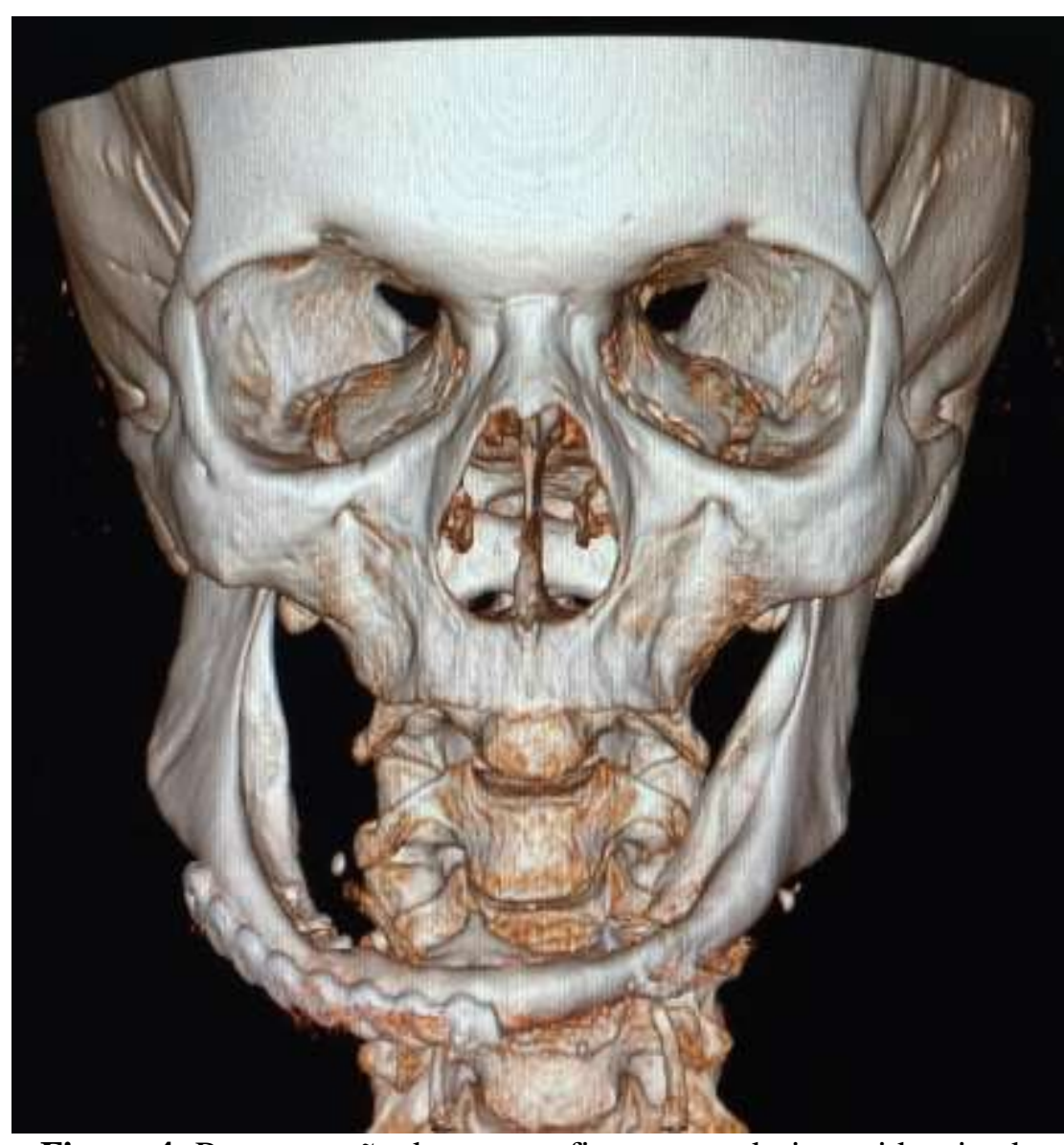

Figura 4: Reconstrução da tomografia computadoriza evidenciando desvio a direita em decorrência do mau posicionamento durante a fixação das fraturas.

O tratamento proposto foi a remoção das fixações existentes, refraturar o osso, restabelecer o perímetro do arco mandibular, fixar as fraturas com uma placa de reconstrução $2.4 \mathrm{~mm}$ e enxertar com osso autógeno. Todo o procedimento foi realizado sob anestesia geral em centro cirúrgico.

Um acesso cervical foi realizado para completa exposição mandibular e remoção das fixações existentes (Figura 5).

Após, a mandíbula foi refraturada, reduzida na posição correta e uma placa do sistema $2.4 \mathrm{~mm}$ RECON foi instalada com 3 parafusos nos ramos e 3 também da sínfise para a fixação das fraturas. Os gaps ósseos foram enxertados 
com osso autógeno triturado da crista ilíaca anterior direita, conforme preconizado por Pereira et al. ${ }^{7}$ (Figura 6). A radiografia panorâmica pós-operatória de 2 meses

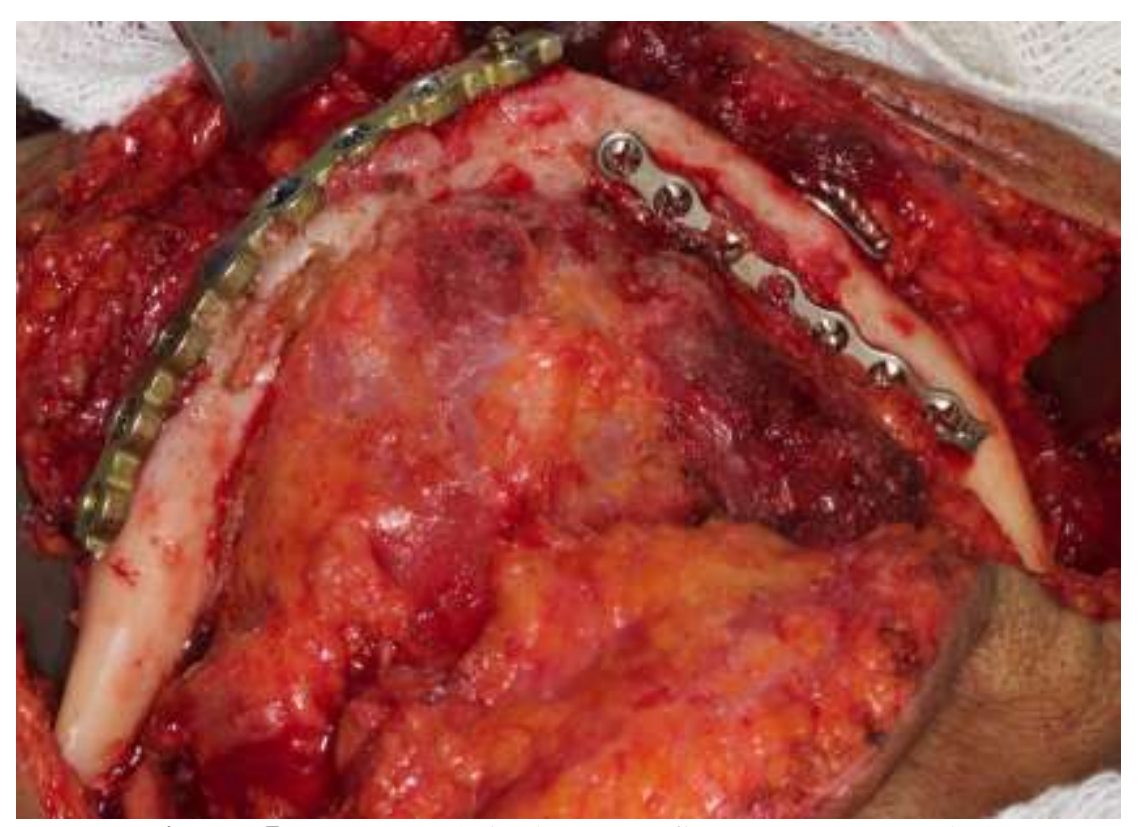

Figura 5: Acesso cervical com as fixações empregadas.

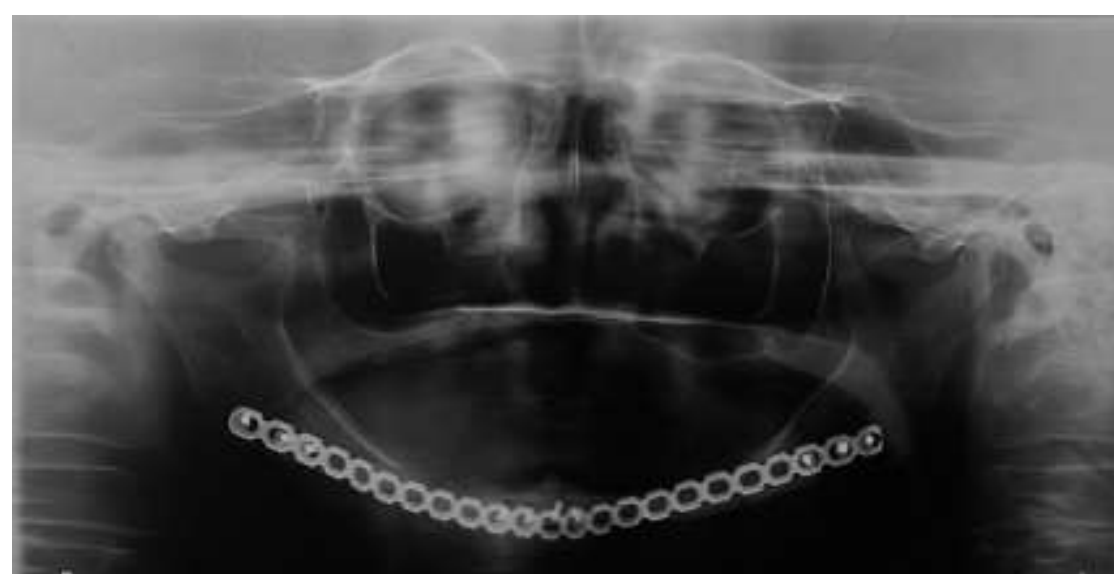

Figura 7: Radiografia panorâmica no pós-operatório de 2 meses demonstrando o melhor posicionamento dos processos condilares.

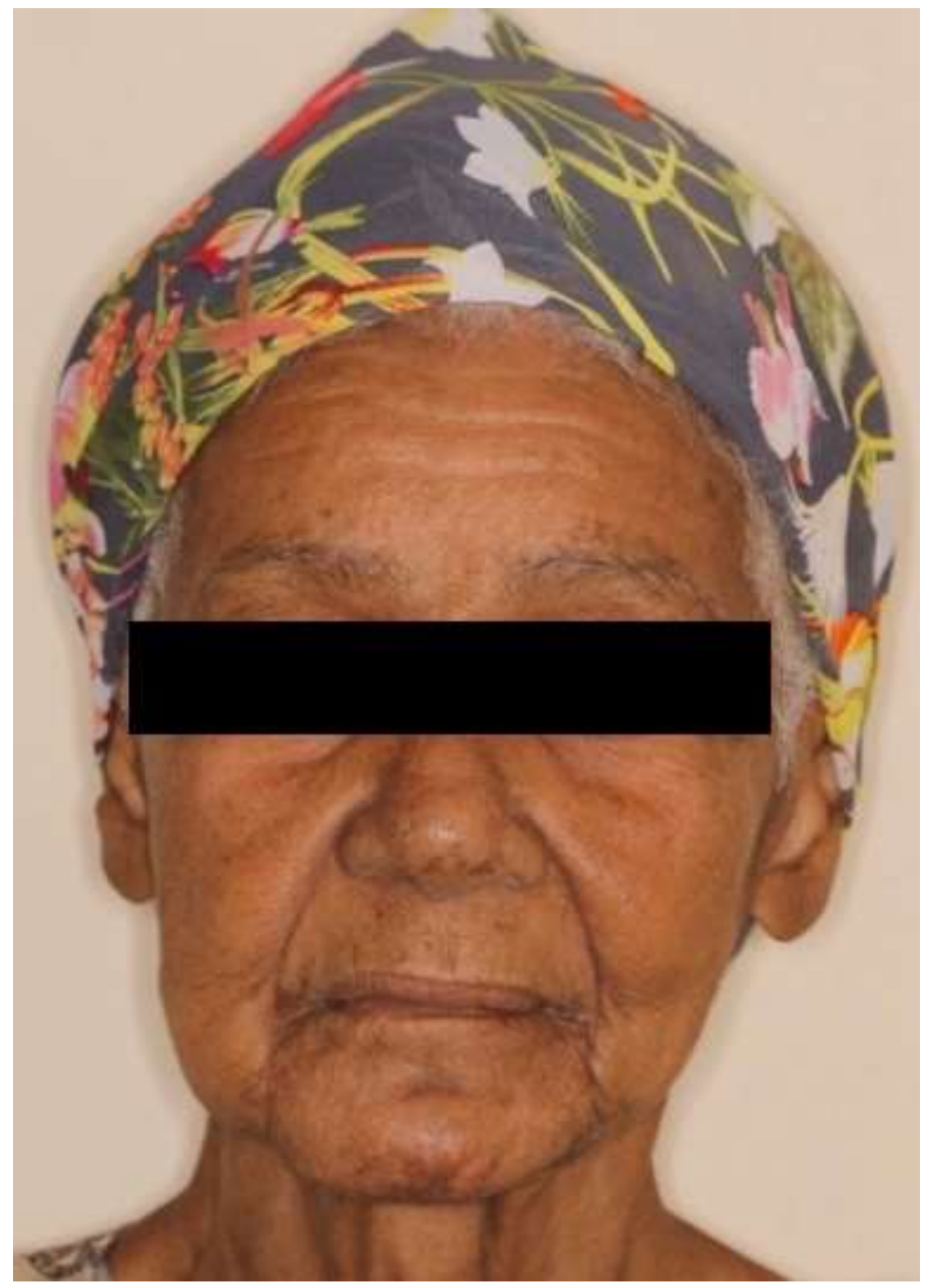

Figura 8: Fotografia clínica frontal evidenciando a correção do desvio mandibular. demonstrou a reposição dos processos condilares e, clinicamente a paciente apresentou melhora do quadro com ausência das queixas álgicas, estéticas e do trismus (Figuras 7 a 9$)$.

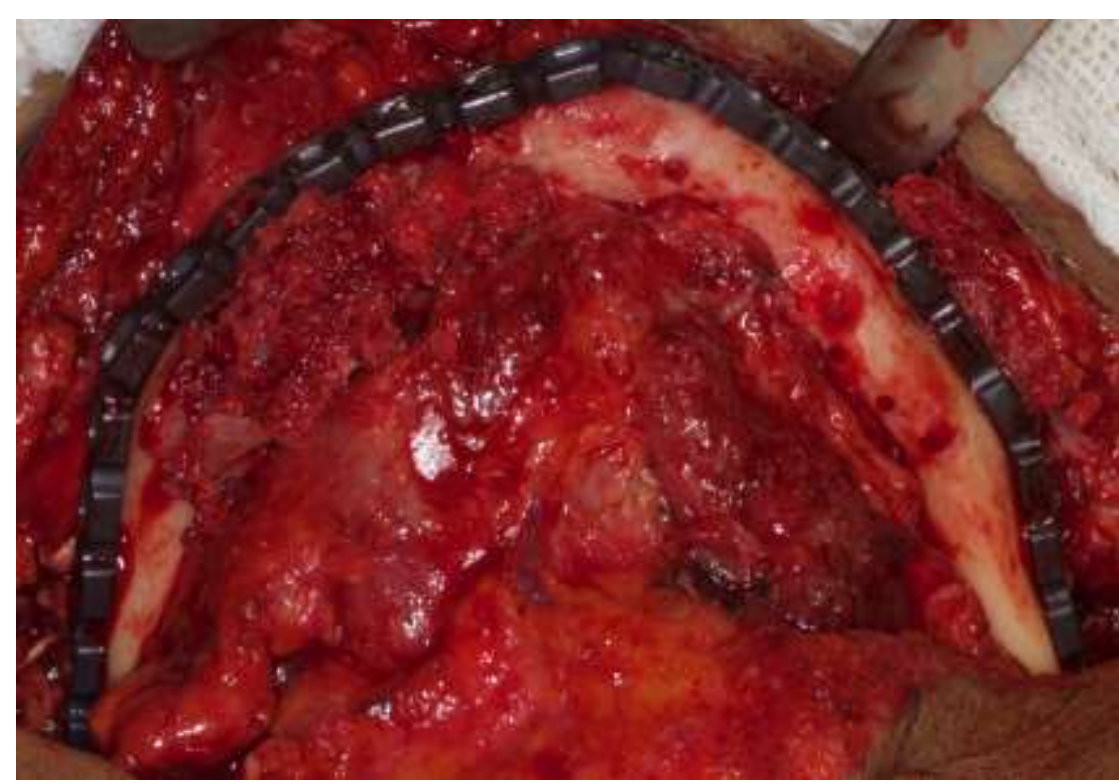

Figura 6: Fase transcirúrgica após a fixação da placa de reconstrução e da enxertia com osso autógeno particulado coletado da crista ilíaca anterior.

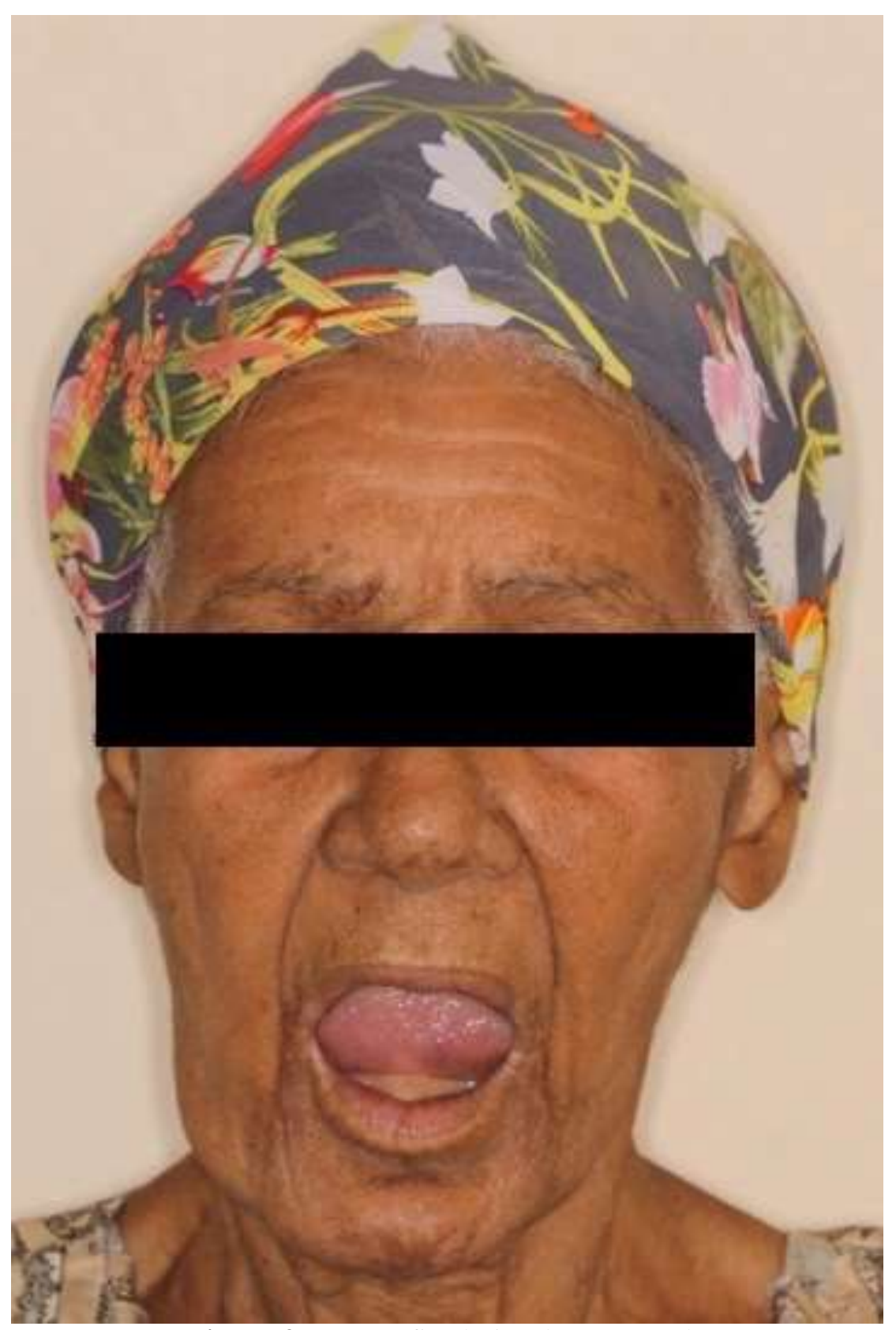

Figura 9: Fotografia clínica demonstrando a ampla abertura bucal da paciente.

Após 1 ano e 7 meses de seguimento pós-operatório, uma nova tomografia foi solicitada e pode-se observar o correto posicionamento dos côndilos mandibulares nas fossas articulares, além do enxerto ósseo integrado (Figuras 10 e 11). 


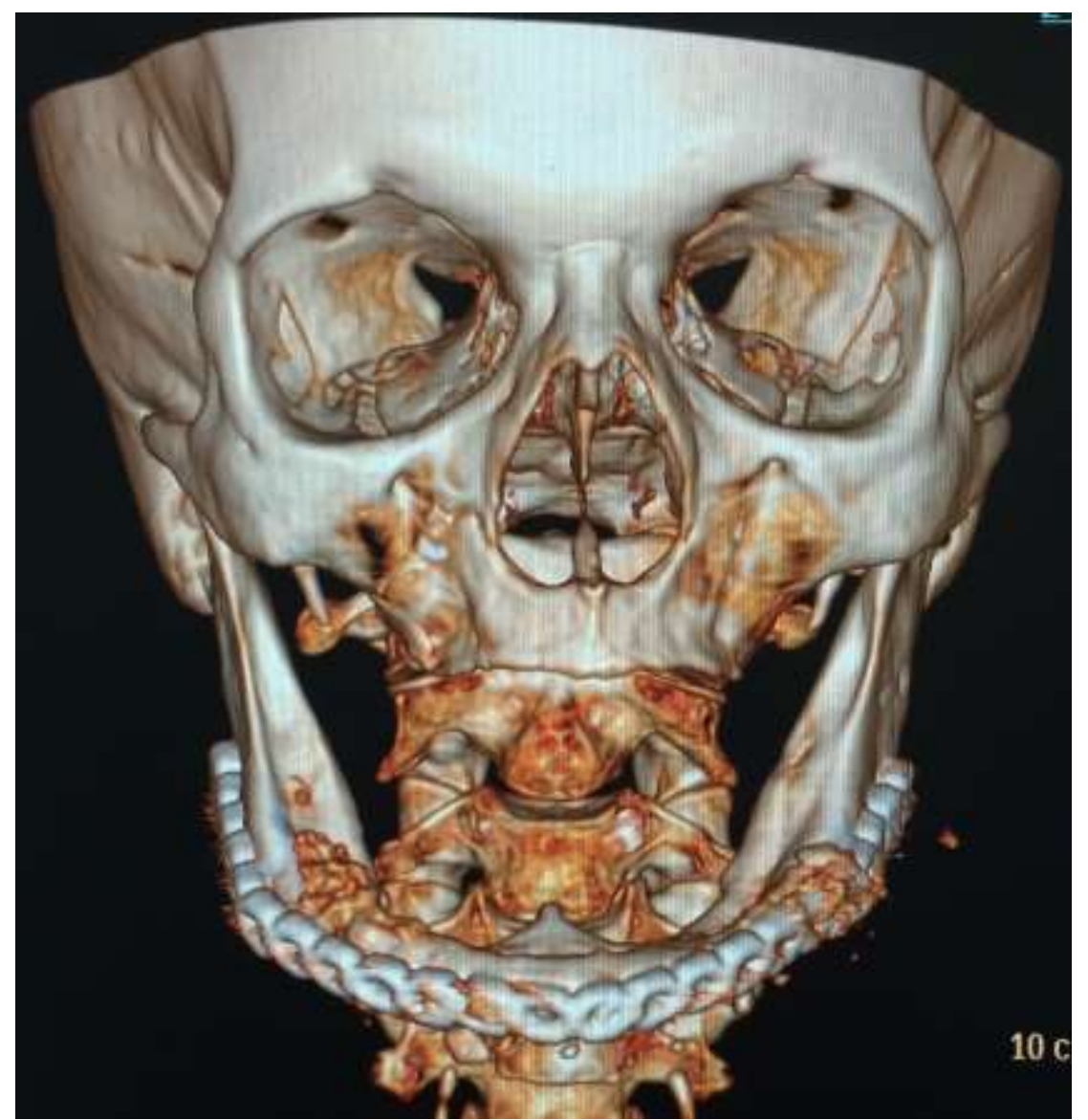

Figura 10: Reconstrução da tomografia computadorizada após a correção das fixações.

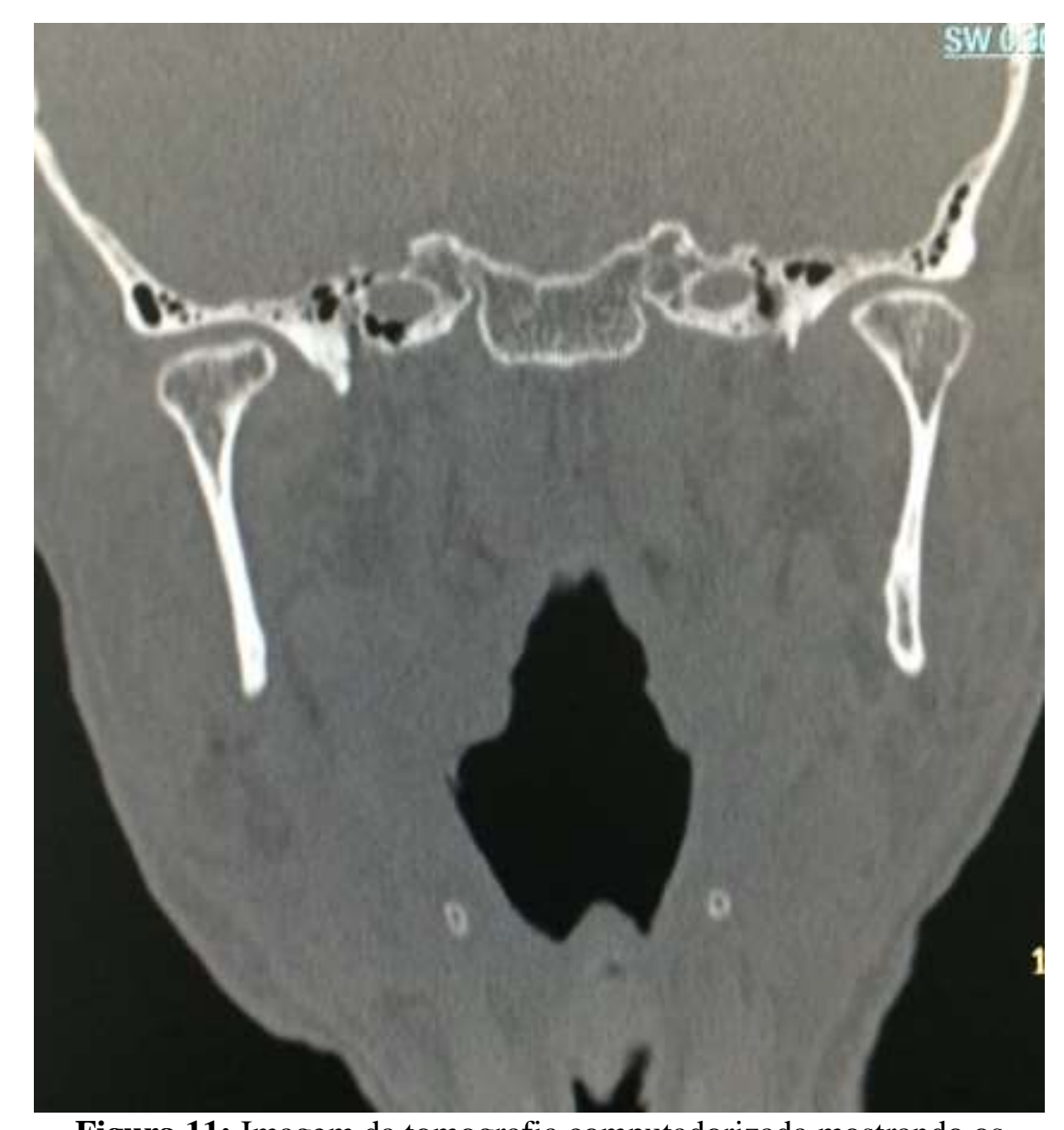

Figura 11: Imagem da tomografia computadorizada mostrando os côndilos mandibulares posicionados corretamente nas fossas articulares.

\section{DISCUSSÃO}

A falta de conhecimento da biomecânica da mandíbula, principalmente nos casos de baixa qualidade óssea, gera complicações como a não-união e infecções decorrentes da mobilidade dos cotos ósseos ${ }^{8}$. Somado a isso, os acessos cirúrgicos conservadores impedem a completa visualização da anatomia do arco mandibular.

A escolha do sistema de fixação no tratamento das fraturas mandibulares parte do princípio do conhecimento de biomecânica do cirurgião. Chiodo e Milles ${ }^{9}$ defendem o uso das miniplacas no tratamento das fraturas mandibulares, contudo, somente quando há segmentos ósseos largos e suficientemente intactos além de, ausência de cominuição.

As mandíbulas atróficas apresentam uma baixa qualidade óssea e também um suprimento vascular reduzido ${ }^{3}$. Fraturas nessas condições não permitem o contato ósseo adequado para que ocorra o reparo ósseo e, na grande maioria dos casos, os pacientes não apresentam condições sistêmicas ideais ${ }^{4}$. Assim, um sistema de fixação mais estável deve ser empregado para que ocorra o suporte necessário frente às cargas mastigatórias atuantes durante todo o processo de reparação.

O sistema "load bearing" preconizado por Spiessl ${ }^{8}$ constitui de uma placa de reconstrução $2.4 \mathrm{~mm}$ que deve ser fixada na borda inferior da mandíbula com os parafusos instalados nas áreas de osso saudável e, de preferência, onde não existam estruturas anatômicas nobres.

O enxerto de osso autógeno é utilizado para o tratamento da não união e má união de fraturas mandibulares bem como nos casos de fraturas de mandíbulas atróficas, pois fornece células osteoblásticas, células mesenquimais indiferenciadas, osteoclastos e fatores de crescimento ${ }^{10,11}$ Seu uso facilita a união óssea, promove mais estabilidade ao osso após o reparo ósseo e permite a possibilidade de restauração protética ${ }^{3}$.

\section{CONCLUSÃO}

O tratamento das fraturas em mandíbulas atróficas é sempre um desafio ao cirurgião maxilofacial. O tratamento conservador pode ocasionar complicações posteriores.

Apesar da natureza agressiva do tratamento proposto, ainda é o melhor e o mais previsível. No entanto, exige do profissional conhecimento da anatomia mandibular, da biomecânica, dos sistemas de fixação e das técnicas cirúrgicas.

\section{REFERÊNCIAS}

1.Kuriakose MA, Fardy M, Sirikumara M, Patton DW, Sugar AW. A comparative review of 266 mandibular fractures with internal fixation using rigid (AO/ASIF) plates or mini-plates. Br J Oral Maxillofac Surg. 1996; 34(4):315-21.

2.Emam HA, Stevens MR. Can an arch bar replace a second lag screw in management of anterior mandibular fractures? J Oral Maxillofac Surg. 2012; 70(2):378-83.

3.Ellis E 3rd, Price C. Treatment protocol for fractures of the atrophic mandible. J Oral Maxillofac Surg. 2008; 66(3):421-35.

4.Luhr HG, Reidick T, Merten HA. Results of treatment of fractures of the atrophic edentulous mandible by compression plating: a retrospective evaluation of 84 consecutive cases. J Oral Maxillofac Surg. 1996; 54(3):250-4.

5.Mugino H, Takagi S, Oya R, Nakamura S, Ikemura K. Miniplate osteosynthesis of fractures of the edentulous mandible. Clin Oral Investig. 2005; 9(4):266-70.

6. Assael LA, Klotch DW, Manson PN, Prein J, Rahn BA, Schili W, et al. Manual of Internal Fixation in the Cranio-Facial Skeleton: Techniques Recommended by the AO/ASIF Maxillofacial Group. New York: Springer; 2014.

7.Pereira R dos S, da Rocha HV Jr, Homsi N, Vieira EH, de Souza FI, Griza GL. Condensed autogenous bone particles: modified technique. J Craniofac Surg. 2015; 26(3):942-3.

8. Spiessl B. Internal Fixation of the Mandible. New York: Springl er-Verlag; 1989. p-223.

9. Chiodo TA, Milles M. Use of monocortical miniplates for the intraoral treatment of mandibular fractures. Atlas Oral Maxillofac Surg Clin North Am. 2009; 17(1):19-25. 
10. Boyne PJ, Upham C. The treatment of long standing bilateral fracture non- and mal-union in atrophic edentulous mandibles. Int J Oral Surg. 1974; 3(5):213-7.

11. Tiwana PS, Abraham MS, Kushner GM, Alpert B. Management of atrophic edentulous mandibular fractures: the case for primary reconstruction with immediate bone grafting. J Oral Maxillofac Surg. 2009; 67(4):882-7.

\section{CONFLITO DE INTERESSES}

Os autores declaram não haver conflitos de interesse.

\section{AUTOR PARA CORRESPONDÊNCIA}

Rodrigo dos Santos Pereira

dr.pereira@live.com

Submetido em 15/12/2016 Aceito em 26/01/2017 